\title{
Experimental Study on Physical Properties of High Quality Recycled Concrete Aggregate
}

\author{
Jun DAI \\ School of Architectural and Civil Engineering \\ Xi'an University of Science and Technology \\ Xi'an, China \\ e-mail: 654578688@qq.com
}

Wenping DU

School of Architectural and Civil Engineering Xi'an University of Science and Technology

Xi'an, China

\author{
Chuanjing LI \\ School of Architectural and Civil Engineering \\ Xi'an University of Science and Technology \\ Xi'an, China \\ e-mail: 657758296@qq.com \\ Qiang YANG \\ School of Architectural and Civil Engineering \\ Xi'an University of Science and Technology \\ Xi'an, China
}

\begin{abstract}
The research beneficiated the concrete waste from two different sites to get the recycled concrete aggregates by the microwave-assisted mechanical beneficiation technology. Then, the author systematically studied the physical properties of recycled coarse aggregates and natural coarse aggregates from both macro and micro perspectives .The results show that: (1) in this experiment, the irradiation time per unit mass is about $5 \mathrm{~min} / \mathrm{kg} \sim 10 \mathrm{~min} / \mathrm{kg}$, and the beneficiation rate is in $30 \%$ $\sim 70 \%$; (2) macroscopic physical experiments show that the recycled coarse aggregates obtained by the microwave-assisted mechanical beneficiation technology is basically the same with natural coarse aggregates in terms of water absorption, apparent density and mass loss., and its physical properties are the most optimal compared with other techniques. (3) from the microscopic point, the scanning electron microscope test shows that the mechanical process poses more damage to the recycled coarse aggregates than the microwave-assisted mechanical beneficiation technology through which the recycled coarse aggregates appears only several micro cracks in the cases after being magnified 5000 times, which is much equal to the natural coarse aggregates. In conclusion, this research validated the high quality of recycled coarse aggregates in the microwave-assisted mechanical beneficiation technology.
\end{abstract}

Keywords-recycled concrete aggregates; high quality; physical properties; scanning electron microscope

\section{INTRODUCTION}

With the development of national economy and the improvement of living standards, annual construction quantities are increasing, which directly leads to the increase of concrete usage. At the same time, large volumes of construction and demolition waste cause many environmental problems, such as the lack of adequate deposition sites, the shortage of natural resources and other issues. In order to meet the healthy and sustainable development of basic construction, a new technology has been proposed and developed. With this technology, new concrete are recreated by the recycled coarse aggregates (RCA) which are beneficiated from the concrete waste from buildings that are torn down. It's an effective method to protect the natural environment. Well, in producing recycled concrete, the first step is the beneficiation of RCA, and the quality of RCA plays a decisive role for the properties of recycled concrete. Therefore, for the wide application of recycled concrete, the performance of recycled coarse aggregate should be studied first. The research work of recycled concrete aggregate is relatively earlier abroad, and the technology is relatively more mature, some countries published the technical standard of recycled concrete. K. Pandurangan et al. [1] analyzed the effects of different treatment methods on the bond strength of recycled aggregate concrete; S. Laserna et al. [2] analyzed the effects of two different types of aggregate on the strength properties of recycled concrete; P. Saravanakumar* et al. [3] analyzed and compared the untreated RCA, natural coarse aggregates (NCA) and treated RCA respectively after $\mathrm{H}_{2} \mathrm{SO}_{4}$ solution, $\mathrm{HNO}_{3}$ solution, $\mathrm{HCI}$ solution and $\mathrm{HCI}+$ silica fume solution. Though the related research started late in China, lots of research results have been obtained after the active exploring of many scholars. Lei Zhu [4] analyzed the distribution law and the dispersion of the main properties of RCA of different sources; Daxing Qian et al. [5] analyzed the regeneration technology of concrete waste, optimized the concrete recycling technology and put forward the method of improving the performance of the recycled concrete. Jianguang Shi [6] analyzed the effect of aggregate composition and grade on concrete strength. The above researches adopt mostly the traditional beneficiation technologies, and quality of RCA beneficiated is generally low. There are two main reasons, firstly the RCA are often attached around by mortar which generates a weak face and affects the quality of recycled coarse aggregate; secondly, the traditional method will make the RCA inner micro cracks increased and weaken the physical and mechanical properties of aggregate. Thus, it's necessary to improve the existent RCA beneficiation technologies.

Combined with the existing results, in this paper, the RCA was beneficiated by the new technology of 
microwave-assisted mechanical beneficiation [7, 8], and was studied of its quality from the macro and micro perspectives. This technology poses a certain degree of damage to the mortar body wrapped with coarse aggregate in the waste concrete, and promotes the stripping of the mortar and the concrete coarse aggregate, so as to realize the efficient and rapid beneficiation. Therefore, the microwave-assisted mechanical beneficiation technology is expected to solve the problem of poor quality of currently beneficiated RCA and realize the beneficiation of high quality recycled concrete.

\section{EXPERIMENT FACILITIES AND MATERIAL PREPARATION}

\section{A. Experimental Facilities}

The microwave device uses the model for the EV923KF6 - NA: household microwave oven. The working parameters: rated voltage is $220 \mathrm{~V}$, rated input power is $1450 \mathrm{~W}$, the output power is $900 \mathrm{~W}$, rated frequency of microwave is $2450 \mathrm{~Hz}$, and the cavity size is $325 \mathrm{~mm} \times 202 \mathrm{~mm} \times 337 \mathrm{~mm}$. The pressure machine adopts NYL-200 type press.

\section{B. Beneficiation of RCA}

Due to the size of the waste concrete block is different, the microwave irradiation can not determine the irradiation time specifically, so we must first find out the best irradiation time of unit mass, in order to avoid additional damage to the coarse aggregate.

The specific test procedures are as follows:

(1) The concrete waste specimens are divided into groups, each group is weighed before drying, and then put into the DZF type vacuum drying cabinet for $24 \mathrm{~h}$ drying, taking out for the second weighing;

(2) The concrete waste specimens are then placed in a microwave oven and irradiated in sequence. The irradiation time is adjusted according to the size of the specimen so that the macroscopic cracks can be seen as a standard when it is removed from the microwave oven, and the law is gradually found out.

(3) Remove it from the microwave oven after irradiation and cool it into the room temperature, then break it down;

(4) Beneficiate the coarse aggregate and weigh its mass, then compared with concrete waste which before beneficiation, and then the beneficiation rate can be obtained.

(5) Acquire the beneficiation rate of recycled coarse aggregate and unit mass's range of the irradiation time according to the above rules.

The analysis and experimental results show that the irradiation time per unit mass is about $5 \mathrm{~min} / \mathrm{kg} \sim 10 \mathrm{~min} / \mathrm{kg}$, and the beneficiation rate is in $30 \%$ to $70 \%$, it provides the reference for using this technology to beneficiate the RCA in the future.

Then using the above experimental results beneficiate the recycled coarse aggregate, the processes are as follows: firstly, weigh the waste concrete block, convert the microwave irradiation time, mechanical crush after microwave irradiation, then get recycled coarse aggregate at last, as shown in Figure1(a).

\section{Material Preparation}

(1) Cement: Select the same batch production of Qinling brand P.O 32.5 composite Portland cement whose apparent density is $310 \mathrm{~kg} / \mathrm{m} 3$.

(2) Sand: The sand are net natural river sand, and the physical and mechanical properties meet the "sand for building" (GB/T14684-2011) requirements, the apparent density is $2262 \mathrm{~kg} / \mathrm{m}^{3}$, the moisture content is $3.9 \%$.

(3) Water: city tap water.

(4) NCA and RCA: Natural coarse aggregate(NCA)are pebble chosen from the Lantian county and particle size of 5 $\mathrm{mm}$ to $40 \mathrm{~mm}$, continuous gradation, smooth surface and grain shape is better.RCA beneficiated by the microwave-assisted mechanical beneficiation technology, As shown in figure 1(a). The RCA from Cehui road and Jianshe road are marked to CRCA and JRCA respectively, and its size is $5 \mathrm{~mm}$ to $40 \mathrm{~mm}$, grading continuously. Two kinds of coarse aggregate are in line with the "ordinary concrete with gravel or gravel quality standards and test methods" (JGJ53-92) requirements. It can be seen from Figure 1 that the surface of the RCA beneficiated by the microwave-assisted mechanical beneficiation technology is much cleaner, compared with other technology, and the aggregate gradation is reasonable. So, the beneficiated RCA has a good quality and meets the test requirements.

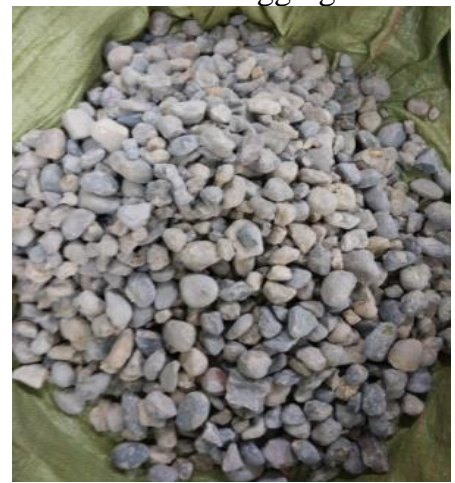

a. RCA beneficiated in the experiment

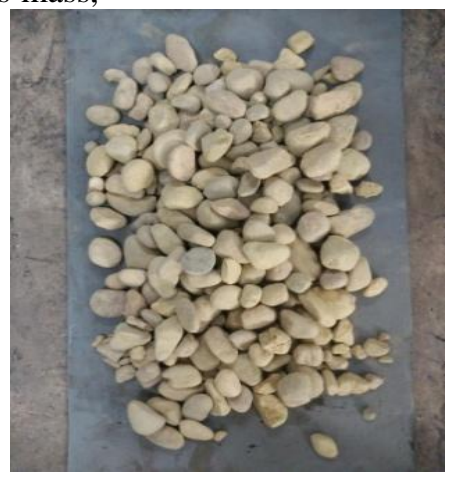

b. NCA

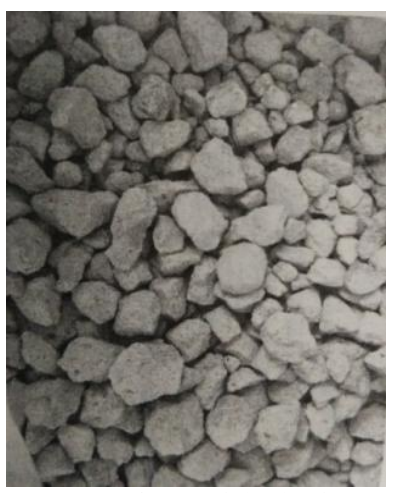

c. RCA after particle shaping

Figure 1. Natural aggregate and recycled coarse aggregate out of different processes 


\section{MAIN PhysiCAL PROPERTIES TEST OF RCA}

The quality of recycled aggregates plays an important role in the performance of RCA. So it is necessary to carry out the experiment of its main physical properties, in order to verify whether the RCA has the characteristic of high quality.

\section{A. Water Absorption Experiment}

For recycled coarse aggregate, water absorption is an important index. It can reflect the damage degree of the microwave-assisted mechanical crushing to RCA. According to the particle size, RCA can be divided into 10 $\mathrm{mm}$ to $20 \mathrm{~mm}, 20 \mathrm{~mm}$ to $30 \mathrm{~mm}, 30 \mathrm{~mm}$ to $40 \mathrm{~mm}$ three groups of water absorption test, as shown in figure 2 . Then, test is carried out in the particle size range the $5 \mathrm{~mm} \sim 40 \mathrm{~mm}$, and compared with the water absorption of NCA in the same grade; the results are shown in Figure 3.

It can be obtained from Figure2 (a) that under the same soaking time; the water absorption rate decreased with the increase of particle size, and that under the condition of the same size, water absorption presented a growth trend along with the increase of immersion time, the growth rate turning smaller gradually, the trend being the same with NCA's. Although the mortar wrapped in the surface of RCA beneficiated by the microwave-assisted mechanical beneficiation technology is less, it will still make a little higher porosity of RCA. Therefore, the water absorption of RCA is still a little greater than that of the NCA.

Figure 2 (b) shows, under the same soaking time, the water absorption of RCA is larger than that of NCA; With the increase of immersion time, the water absorption of both RCA and NCA are presenting increasing trends; the water absorption of CRCA and JRCA is similar and the growth trend is the same; the water absorption of RCA is much closer to that of NCA in this experiment. Compared to the minimum water absorption of $\mathrm{He} \mathrm{YE} \mathrm{[9]:} \mathrm{10min} \mathrm{water}$ absorption of $1.58 \%, 30 \mathrm{~min}$ water absorption of $1.74 \%, 24 \mathrm{~h}$ water absorption of $1.82 \%$, the results of this experiment are much smaller. In contrast to the results obtained by other technologies, the water absorption of RCA in the experiment is much closer to that of NCA. The experimental results show that the amount of mortar attached to the surface of the RCA is relatively less, and the porosity is relatively low, thus indirectly explaining the superiority of the microwave-assisted mechanical beneficiation technology, the higher quality of RCA.
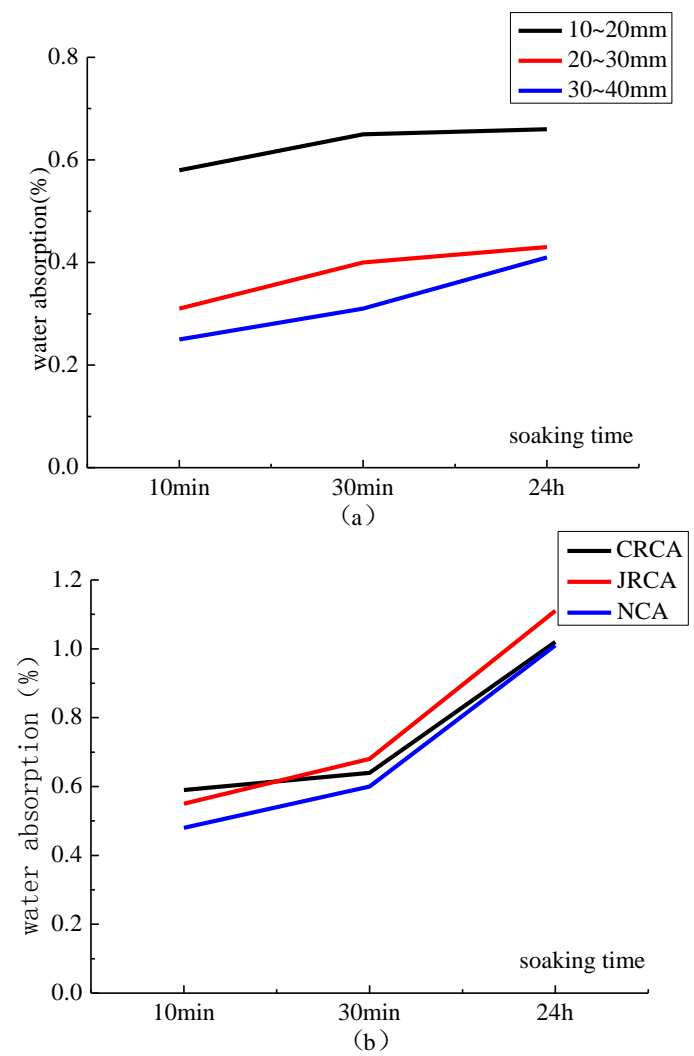

Figure 2. The water absorption of coarse aggregate in different soaking time

\section{B. Apparent Density Experiment}

According to the "performance of ordinary concrete mixture test method" (GB/T50080-2011), the RCA particles of less than $5 \mathrm{~mm}$ are screened and divided by the quarter method to not less than $2 \mathrm{~kg}$. After rinsing, the mixture is divided into two spares. The 2019.1g weight of RCA is divided into two times to pack into $1000 \mathrm{ml}$ wide mouth bottle; the sand is divided into two parts, respectively $300 \mathrm{~g}$; the results are shown in Table 1.

As shown in Table 1, the apparent density of NCA is $2850 \mathrm{~kg} / \mathrm{m}^{3}$; the apparent density of JRCA is $2442 \mathrm{~kg} / \mathrm{m}^{3}$, which is $85.68 \%$ of NCA, $14.32 \%$ lower, and the apparent density of JRCA is $2467 \mathrm{~kg} / \mathrm{m}^{3}, 86.56 \%$ of $\mathrm{NCA}, 13.44 \%$ lower. Although there is less mortar on the surface of the RCA, the surface still has a certain amount of mortar, which leads to the decrease of its apparent density. Compared with other processes, the apparent density of recycled coarse aggregate after particle shaping was $2530 \mathrm{~kg} / \mathrm{m}^{3}$, which was $3.6 \%$ and $2.55 \%$ higher than JRCA and CRCA, respectively, which was $5.11 \%$ higher than that of untreated coarse aggregate. In contrast to the existing research results, the apparent density of this experiment is slightly larger.

TABLE I. APPARENT DENSITY OF NCA AND RCA

\begin{tabular}{cccc}
\hline Aggregate name & NCA & JRCA & CRCA \\
\hline Apparent density $\left(\mathrm{kg} / \mathrm{m}^{3}\right)$ & 2850 & 2442 & 2467 \\
\hline
\end{tabular}




\section{Aggregate Solidity Experiment}

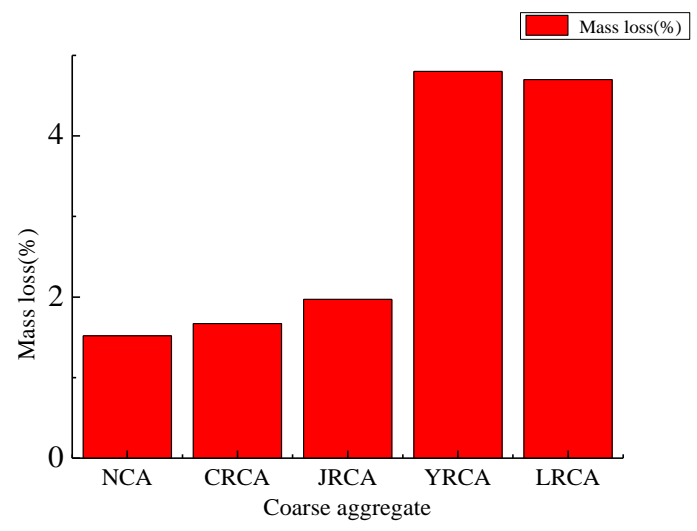

Figure 3. Mass loss of coarse aggregate

Aggregate solidity refers to the durability of aggregate against climate or other physical effects, reflecting the ability of aggregate to resist the damage caused by climate change or other factors. So the solidity of aggregate is also a major factor in characterizing its performance, it is
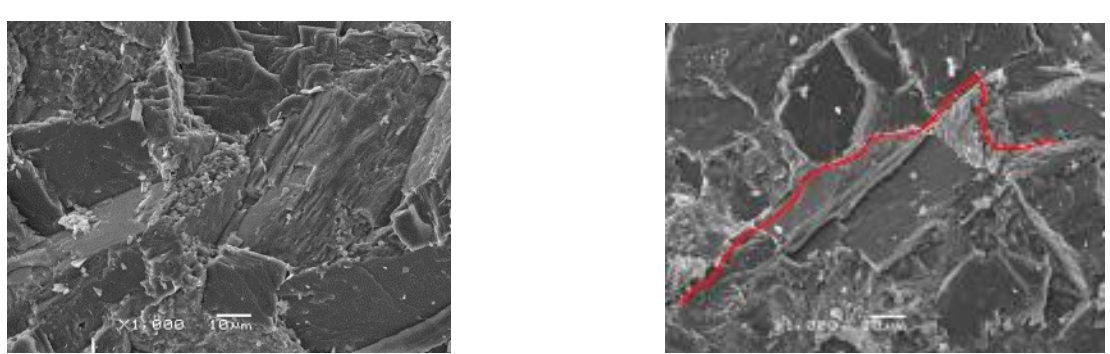

cessary to measure them. Since the RCA beneficiated by the microwave-assisted mechanical beneficiation technology used in this test contains less mortar, the solidity of CRCA, JRCA, NCA and RCA beneficiated by other processes are measured. The results of the solidity test of the RCA are shown in Fig.3.

Figure 3 shows the mass loss of NCA was $1.52 \%$, the mass loss of CRCA was $1.67 \%$, the mass loss of JRCA was $1.97 \%$, the mass loss of He Ye (YRCA) was $4.8 \%$, which was 2.87times than that of CRCA, 2.44times than that of JRCA. The results of this experiment are $64.47 \%$ and $58.09 \%$ less than those of Zhanyin Li [10] (LRCA).The mass loss he obtained is $4.7 \%$, which is 2.81 times of CRCA and 2.39 times of JRCA. The results of the test were respectively reduced by $65.21 \%$ and $58.96 \%$ compared with the results of He YE. The mass loss of CRCA increased by only $9.87 \%$ and that of JRCA increased by $29.61 \%$,compared with the NCA. Therefore, it can be seen that the RCA beneficiated in this experiment is more durable than other recycled coarse aggregates.

\section{The SCANNING EleCtron Microscope EXPERIMENT}

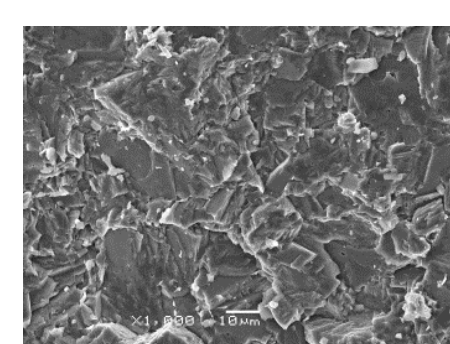

NCA

RCA beneficiated by the mechanical crushing

RCA beneficiated by the microwave-assisted mechanical technology

(a) Electron microscope scanning images magnified 1000 times
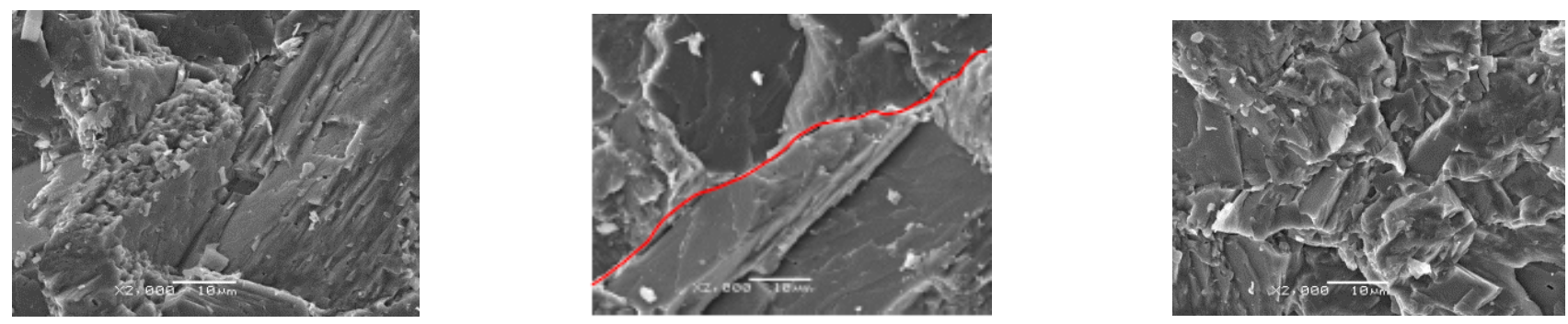

NCA

RCA beneficiated by the mechanical crushing

RCA beneficiated by the microwave-assisted mechanical technology

(b) Electron microscope scanning images magnified 2000 times
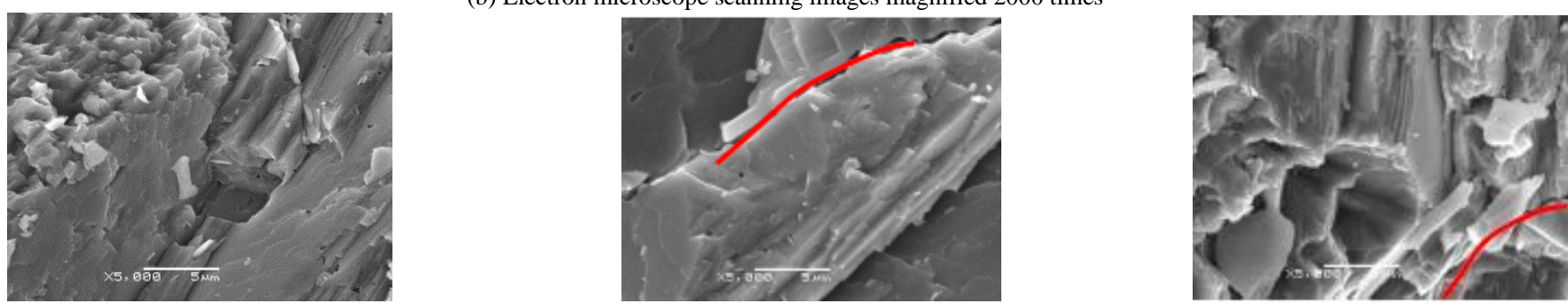

NCA

RCA beneficiated by the mechanical crushing

RCA beneficiated by the microwave-assisted mechanical

(c) Electron microscope scanning images magnified 5000 times

Figure 4. SEM images with different magnification 
The former experiments only studied mechanical properties of the RCA from the macroscopic perspective, and they were similar to that of the natural aggregate. But the same can not be said for the mesoscopic study. Therefore, this experiment studied the performance of RCA from the micro-perspective and further verified the high quality of RCA which beneficiated by the microwave-assisted mechanical technology. The test materials were selected from NCA, RCA beneficiated by the mechanical crushing and RCA beneficiated by the microwave-assisted mechanical technology. The SEM images of different magnifications were shown in the following Figure 4.

From Fig.4, it can be concluded that the changes of the micro-cracks in the RCA beneficiated by the mechanical crushing are obvious among the three groups, and there is a continuous crack in the process from 1000 to 5000 times magnification which takes a large proportion. During the process of natural coarse aggregate changing from 1000 times to 5000 times, there is only a small amount of tiny cracks in the RCA, and the proportion of the cracks is very small which is almost negligible. Micro cracks can be observed only under the condition of magnification of 5000, and the number of micro cracks is nearly the same as that NCA. It can be concluded that the microwave-assisted process is much less damage to the RCA than the mechanical crushing process, which is worthy of popularization.

\section{CONCLUSION}

In this paper, the experimental research method is adopted, and the concrete waste is treated by the microwave-assisted mechanical beneficiation technology. The RCA is obtained, and its mechanical properties are studied both macroscopically and microscopically. The following conclusions are obtained:

(1) The experimental results show that the irradiation time is about $5 \mathrm{~min} / \mathrm{kg} \sim 10 \mathrm{~min} / \mathrm{kg}$, and the sampling rate is between $30 \% \sim 70 \%$, which can be used to provide a reference for the beneficiation of RCA.

(2) Through the macroscopic physical experiment, under the condition of continuous grading in the same particle size range, the water absorption rate of the recycled coarse aggregate is slightly larger than that of the natural coarse aggregate; its apparent density is lower than natural coarse aggregate; its mass loss is slightly larger than that of NCA; compared with other techniques, the physical properties of RCA obtained by the microwave-assisted mechanical beneficiation technology is optimal.

(3) Through the scanning electron microscope experiment, it concluded that the damage degree of RCA by mechanical crushing is large from the microscopic perspective, and that RCA beneficiated by microwave-assisted mechanical technique whose micro-cracks are only shown under the condition of magnification of 5000, is closer to the NCA.

(4) Based on the results of both macroscopic and microscopic experiments, it can be seen that the microwave-assisted mechanical beneficiation technology greatly reduces the amount of mortar wrapped on the coarse aggregate surface, reduces the damage to the RCA, and improves the physical properties of RCA. The experimental results show that the high quality of the RCA by the microwave-assisted mechanical beneficiation technology is verified.

\section{ACKNOWLEDGMENT}

The authors thankfully acknowledge the support of the Natural Science Foundation of China (NO.51174159), School of Architectural and Civil Engineering Xi'an University of Science and Technology.

\section{REFERENCES}

[1] K. Pandurangan, A. Dayanithy, S. Om Prakash, "Influence of treatment methods on the bond strength of recycled aggregate concrete," Construction and Building Materials, 2016 (120), pp.212-221.

[2] S. Laserna, J. Montero, "Influence of natural aggregates typology on recycled concrete strength," Construction and Building Materials, 2016(115), pp. 78-86.

[3] P Saravanakumar*, K. Abhiram, B Manoj, "Properties of treated recycled aggregates and its influence on concrete strength characteristics, "Construction and Building Materials, 2016(111), pp. 611-617.

[4] Zhu Lei. "Research on asessment of recycled coarse aggregates and early performance of Recycled Concrete", Nanjing: Nanjing University of Aeronautics and Astronautics, 2011.

[5] Qian Da-hang, Wang Jia-yang, "Effect study on recycled concrete performance by optimizing regeneration process,"Construction Technology, 2015, 44(12), pp. 69-72.

[6] Dan Jian-guang., "Experimental research and computational analysis of recycled aggregate concrete performance," Shanghai: Shanghai University, 2010.

[7] Dai Jun, Wang Qian. "Preliminary studies on new beneficiation technique of recycled concrete aggregate," Concrete, 2014(8), pp. 133-135.

[8] Chen Xin-nian, Dai Jun, Meng Zhen, "Microwave-assisted mechanic technigue of improving the quality of recycled concrete aggregates," Journal of Xi'an University of Science and Technology, 2013, 33(5), pp. 604-608.

[9] Ye He, "Mechanical properties and durability of concrete with high quality recycled concrete aggregate," Hangzhou: Zhejiang University of Technology, 2009.

[10] Li Zhan-yin, "Experimental studies on performance of the recycled aggregate concrete," Xi'an: Xi'an University of Architecture and Technology, 2003. 